\title{
Quark Gluon Plasma
}

\author{
Nuclear Research with Ultrarelativistic Heavy lons
}

\author{
Rainer Santo, Münster
}

(Institut für Kernphysik, Universität Münster)

\begin{abstract}
Nuclear matter is known to consist of nucleons (neutrons and protons) which are bound together by the strong nuclear forces. Over many years, nuclear physics has concentrated on the study of nuclear matter by investigating nuclear reactions and nuclear excitations close to the ground state. The interest in probing nuclear matter at extreme conditions has arisen only in the last years and has rather different sources. On the one side, experiments performed with the $1 \mathrm{GeV} / \mathrm{A}$ heavy ion beam of the Bevalac at Berkeley have demonstrated that nuclear matter can appreciably be compressed and excited, displaying features well described in hydrodynamical models. On the other, elementary particle spectroscopy and collisions at high energies have provided convincing evidence for the quark model of hadrons, according to which nucleons are no longer elementary but consist of three quarks bound together within the nucleon radius (of about $1 \mathrm{fm}$ ). Within the current theory of strong interactions, Quantum Chromodynamics (QCD), quarks are said to be confined to their bags and interact via gluon exchange.
\end{abstract}

The immediate question in the context of nuclear matter is, of course: what happens if nuclear matter is more and more compressed and the quark bags begin to overlap? Intuitively one would expect a gradual dissolving of the bags so that quarks no longer remember their original nucleons but begin to form an extended system of quarks and gluons. Unfortunately, standard QCD cannot give an immediate answer to this question. The pecularities of the quark interaction, which is weak at small distances but increases tremendously at larger distances, allow analytical solutions only in the regime of the so called "asymptotic freedom", i.e. at small distances or at very high energies. For the range of some $\mathrm{fm}$, which is relevant for binding nucleons within nuclei, the magnitude of the strong interaction force prevents a perturbative treatment of QCD. (It seems that this inability is one of the reasons why nuclear and par- ticle physics have developed so differently over the last decades).

The introduction of lattice QCD by $K$. Wilson (Nobel Prize 1982), however, has opened the possibility of treating strong interacting quark gluon systems by a statistical method. Various calculations within this recipe have been performed in the meantime and have, indeed, shown that a phase transition from ordinary nuclear matter to quark matter is expected to occur at finite values of energy density $\varepsilon$ and temperature $T$. This new state of matter, which should be formed at sufficiently high compression is often named a "quark gluon plasma", since it resembles the transition from neutral atomic matter to an ionized plasma. While in the latter case, increasing pressure produces the wellknown Debye screening of charges, leading finally to free moving electrons, the increasing pressure on the quark bags liberates quarks and generates "colour conductivity". Compared with attempts to isolate single quarks or to study their quasi free interaction at very high energies, the investigation of a possible phase transition offers an attractive alternative possibility for testing QCD, in particular, in the regime relevant to nuclear physics.

A search for the quark gluon plasma is, however, also important for cosmology questions relating to the early Universe. According to current models the Universe may, in the very first moments, have consisted of free quarks and gluons, which subsequently have condensed to the normal hadronic matter of today. Attempts to create the quark gluon plasma in the laboratory by a "little bang" will therefore shed some light on the "Big Bang".

According to lattice $Q C D$ calculations, the critical values for the phase transition should be $\varepsilon_{\text {crit }}=2-5 \mathrm{GeV} / \mathrm{fm}^{3}$ and $T_{\text {crit }}=200-300 \mathrm{MeV}$. In a laboratory experiment, the only way of producing such energy densities over a large volume of nuclear matter is through a violent interaction of two heavy nuclei. Several questions, however, immediate- ly arise: What is the projectile energy required and what fraction of this energy is actually converted into internal energy in a thermodynamical sense?

(This question of "nuclear stopping power" also indicates, why pp collisions are not very useful in this context, since the energy there is mainly concentrated in leading particles and jets; or in other words: enough energy but too little entropy is produced).

Even if the critical energy is reached, the question arises, how the formation of the quark gluon plasma can unambiguously be identified. A number of characteristic signals have been discussed in the last years, but it has turned out, that the numerical values often depend on the dynamics of the reaction which is not well known. It is clear that in a first step the longitudinal energy of the projectile has to be converted into undirected thermal energy of the compressed reaction zone which eventually forms the plasma. Cooling may proceed then by photon and particle emission or by thermodynamic expansion. In any case - with or without quark gluon plasma formation - the system will finally decay mainly into hadrons. Characteristic signals therefore consist not of new particles or resonances but of an enhancement or depression of yields. Determining these requires particularly precise experiments with reliable calibrations.

The signals emerging directly from the plasma with very little distortion come from virtual and real photons. An enhancement of those so called "direct" photons or lepton pairs is predicted at the transition, but is very hard to measure in the presence of a tremendously high hadronic background.

Another signal is provided by the yield of strange particles compared to nonstrange particles, which is also predicted to be enhanced. The theoretical predictions, however, seem to have changed very drastically over the last several years.

A very spectacular prediction has been made recently in connection with 

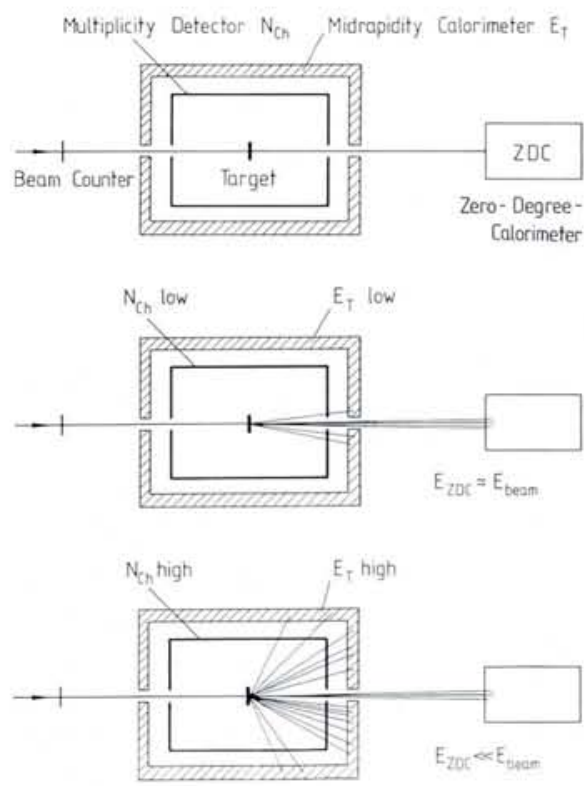

Fig. 1 - a) Schematic sketch of experimental arrangement;

b) Typical pattern for a peripheral reaction or high nuclear transparency;

c) Typical pattern for a central collision with large stopping.

the $\mathrm{J} / \psi$ particle. Here a strong depression of the yields is predicted for the plasma state due to the melting of the quark bags which bind the $c \bar{c}$ pair.

Since, as mentioned above, all of these signals are difficult to identify on an absolute scale, they have to be measured as a function of various parameters and, most importantly, in comparison with data on $\mathrm{p}$-nucleus and pp collisions.

\section{CERN Experiments}

To attack this problem of phase transitions of nuclear matter in heavy ion reactions, a number of experiments were designed and performed during 1986 and 1987 with the ${ }^{16} \mathrm{O}$ and ${ }^{32} \mathrm{~S}$ beam of the SPS at CERN. The acceleration of heavy ions in the SPS had become possible by a joint effort of the Gesellschaft für Schwerionenforschung, Darmstadt (GSI), which provided the special heavy ion source, and the Lawrence Berkeley Lab., Berkeley (LBL) which built the preacceleration system. Using a very minimum of machine time for development, the CERN accelerator group was able to deliver a beam to the various experimental areas with an efficiency of about $80 \%$. The energy used in the experiments was $60 \mathrm{GeV} / \mathrm{A}$ and $200 \mathrm{GeV} / \mathrm{A}$. For ${ }^{32} \mathrm{~S}$ nuclei this amounts to a world record of $6.4 \mathrm{TeV}$ total energy.

Since these experimental conditions opened a completely new field, the first exploratory experiments had the difficult task of covering the expected phy- sics as completely as possible, whilst at the same time remaining open and sensitive to the unknown. Since high energy density is a mandatory pre-requisite of a phase transition, the experiments had to take care of measuring the relevant global observables triggered for the most violent central collisions. The scheme of such measurements is sketched in Fig. 1. The incoming ion is monitored in a beam counter, hits the target and is absorbed down-stream in a so called zerodegree-calorimeter (ZDC). The target region is surrounded by a (more or less) complete arrangement of calorimeters (mid-rapidity calorimeters) and multiplicity detectors.

The observables measured are primarily the transverse energy $E_{\mathrm{T}}$ or $d E_{\mathrm{T}} / d y$ and the charged particle multiplicity $N_{c h}$ or $d N_{c h} / d y$. The rapidity variable $y$ is a relativistically convenient measure of the velocity. Transformation to another Lorentz system amounts only to adding rapidities, so that rapidity distributions remain unchanged. At very high energies, $y$ defined as

$$
y=1 / 2 \ln \left(E+P_{\|}\right) /\left(E-P_{\|}\right)
$$

will depend on the angle $\theta$ only and transform as $y \rightarrow \eta=-\ln \operatorname{tg} \theta / 2$ called "pseudorapidity". $P_{\|}$is the momentum parallel to the beam. The transverse energy $E_{\mathrm{T}}$ is defined as $E \sin \theta$ with $E$ and $\theta$ mesured in the calorimeters.

Analogous to $E_{\mathrm{T}}$ the transverse momentum $p_{T}$ can be defined, which is a relativistically invariant quantity. For $200 \mathrm{GeV} / \mathrm{A}{ }^{16} \mathrm{O}$ nuclei the rapidities $y$ range from $y=0$ (target rapidity) to $y=6$ (projectile rapidity). Since the most central events are expected to occur at $y \approx 3$ (mid-rapidity) most detectors are placed at this region.

Event type characterisation by this set-up is sketched in Fig. 1 b and c. In b) the projectile suffers little interaction in the target, so $E_{\mathrm{ZDC}}$ is close to $E_{\text {beam }}$ and, at the same time, $E_{\mathrm{T}}$ and $N_{\mathrm{ch}}$ are small. This will apply to peripheral reactions (large impact parameter); it alternatively may also indicate, however, a high degree of transparency in a central collision. In c) the situation is sketched for a violent central collision, where most of the projectile energy is absorbed in the target nucleus. Consequently, $E_{\mathrm{ZDC}}$ is small compared with $E_{\text {beam }}$ but $E_{\mathrm{T}}$ and $N_{\text {ch }}$ become larger and allow an estimate of the achieved energy density.

All three experiments, WA80, NA35, NA34/2 use set-ups following the above scheme with somewhat different technical solutions, although each has some special additional devices to look for the characteristic signals from the hot and compressed reaction zone.
The WA80 Collaboration of GS Darmstadt, LBL Berkeley, ORNL Oak Ridge, Lund, Münster, (Spokesman H.H. Gutbrod) uses a ZDC with an opening angle of $\pm 0.3^{\circ}$, a mid-rapidity calorimeter $(2.4 \leq \eta \leq 5.5)$ and large streamer tube arrays for multiplicity measurements. Together with the Plastic Ball (transferred to CERN from the Bevalac) these arrays provide a nearly complete coverage for multiplicity measurements. In the backward hemisphere, the Plastic Ball also serves as a spectrometer to study the target fragmentation.

To look for exclusive reaction channels a finely granulated lead glass photon detector of 1278 modules is used at mid-rapidity. It allows precise measurement of $\pi^{\circ}$ and $\eta^{\circ}$-spectra which give information on the thermodynamical behaviour of the system or the onset of hard QCD processes. The main goal, however, is the measurement of direct photons, which are one of the most promising signals.

The NA35 Collaboration of Athens, Bari, CERN, Cracow, GSI Darmstadt, Frankfurt, Freiburg, Heidelberg, LBL Berkeley, Marburg, MPI München, Warszawa, Zagreb, (Spokesman R. Stock) uses as central detector a large streamer chamber with photographic readout. Apart from measuring spectra of charged particles, this device serves at the same time as a multiplicity detector. The main virtue of the streamer chamber is, however, the capability of detecting rare events. As mentioned before, the production of strange particles like $\Lambda$ and $\bar{\Lambda}$

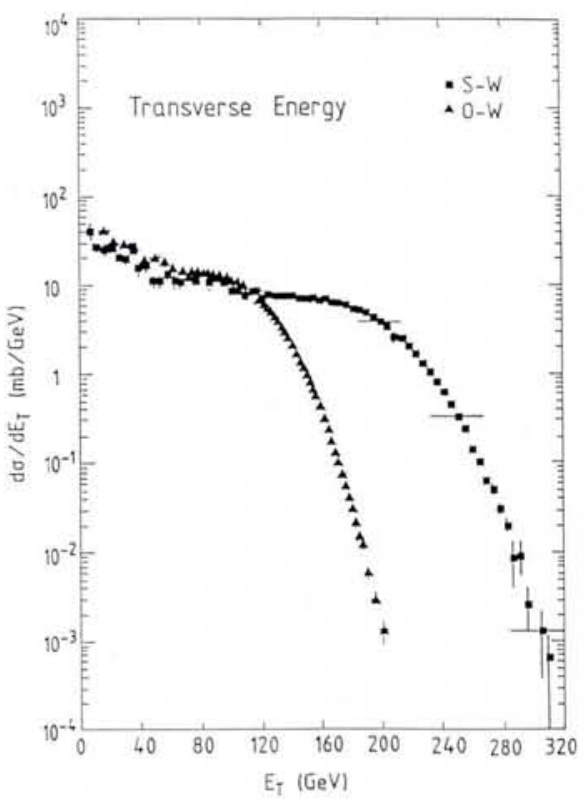

Fig. 2 - Transverse energy spectra for ${ }^{16} \mathrm{O}+W$ and ${ }^{32} \mathrm{~S}+W$ at $200 \mathrm{GeV} / \mathrm{A}$ measured by the NA34/ 2 collaboration. Note that with the larger ${ }^{32} S$ projectile $E_{T}$ extends to larger values. 
particles is of particular interest and is intensively investigated.

Additional event characterisation is achieved by a zero degree as well a midrapidity calorimeter and a finely segmented photon position detector.

The NA34/2 Collaboration, a large international collaboration of 26 institutions (Spokesman H. Specht) has concentrated on a nearly complete calorimetric coverage of the whole rapidity region $(-0.2 \leq \eta \leq 2.9)$. As an example, transverse energy distributions are shown in Fig. 2, (which are also in good agreement with WA 80 and NA35 data in a smaller $\eta$-range). Multiplicities are measured by silicon strip detectors. In a small window, charged particle spectra with particle identification are measured by a magnetic spectrometer. Downstream, NA34/2 has installed a large myon-spectrometer instead of a zero degree calorimeter. Dimyon detection is one of the interesting issues in connection with the phase transition.

The NA38 Collaboration of Annecy, CERN, Clermont-Ferrand, Palaiseau, Lisboa, Lyon, Paris-Sud/Orsay, Strasbourg and Valencia (Spokesman L. Kluberg) has completely concentrated on the dimyon measurement in the region of the $\mathrm{J} / \psi$ resonance using a setup from the former NA10 experiment.

\section{Results}

A large fraction of the 1986 and 1987 runs have now been processed and a number of interesting results have emerged and have partially been published. The most encouraging result from the global measurements is the observation that nuclei, even at these high energies of $200 \mathrm{GeV} / \mathrm{A}$, are not transparent but still show a large amount of stopping. This is seen e.g. by the value of $E_{\mathrm{T}}^{\max }$ increasing with target mass. Multiplicity measurements show a completely parallel behaviour. In this context it should be noticed that multiplicities up to 500 charged particles have been observed showing the large entropy production in heavy ion reactions. Complementary information is provided by the ZDS calorimeters used in NA35 and WA80. All three observables: $E_{\mathrm{T}}, E_{\mathrm{ZDC}}$ and $N_{\mathrm{ch}}$ are found to be strongly correlated so that each of these quantities can be used for selecting different event classes (Fig. 3).

From the $E_{\mathrm{T}}$ distributions (e.g., Fig. 2) a first estimate can be made for the energy densities although there is no generally accepted method for extracting these quantities. Usually, the formula derived by Bjorken is applied:

\section{NORDITA, COPENHAGEN}

\section{ASSISTANT PROFESSOR IN THEORETICAL ASTROPHYSICS or PARTICLE PHYSICS}

NORDITA, the Nordic theoretical physics institute, located at the Niels Bohr Institute of Copenhagen University, has an opening for an assistant professor in theoretical astrophysics or particle physics, starting in September 1989, or later if that is more convenient.

The successful applicant is expected to guide fellows at roughly the postdoctoral level and to interact with colleagues at NORDITA and elsewhere in the Nordic countries. The position provides excellent opportunities to pursue original research and to have contact with a wide range of developments in theoretical physics. There are good facilities for travelling to other institutes and to meetings outside the Nordic countries; the assistant professor will be encouraged to invite guest scientists to NORDITA.

The initial appointment will be for three years, with the possibility of renewal up to a total of six years. The annual salary will be in the range of 220.000 - 300.00 Danish Kroner depending on experience.

Those interested in the appointment should send a curriculum vitae, a list of publications and the names of three referees before 15 December 1988 to the:

Director, Nordita, Blegdamsvej 17, DK-2100 Copenhagen $\emptyset$, Denmark.

Telephone: +45 (1) 421616 bitnet: NRN @ NBIVAX.NBI.DK Telefax: +45 (1) 389157

There is no restriction on the nationality of the applicant. Those wishing to recommend suitable candidates are urged to contact the Director.

$$
\varepsilon=\left(1 / \tau_{\mathrm{o}} \pi R_{\mathrm{p}}^{2}\right)\left(d E_{\mathrm{T}} / d y\right)
$$

where $d E_{\mathrm{T}} / d y$ is usually replaced by the value of $d E_{\mathrm{T}} / d \eta$ experimentally determined (for a certain $\eta$ range). $\pi R_{\mathrm{p}}^{2}$ is the area of the interaction zone and, in the case of a small $\mathrm{O}$ or $\mathrm{S}$ projectile encountering a heavy nucleus, will be the radius of the projectile. The most uncertain number - which is not directly accesible experimentally - is the formation time $\tau_{0}$, which is normally taken as $1 \mathrm{fm} / c$, but other numbers varying within factors of 2 are also discussed. For central collisions of $200 \mathrm{GeV} / \mathrm{A},{ }^{16} \mathrm{O}$ projectiles on a heavy target, and using $\tau_{\mathrm{o}}=1 \mathrm{fm} / c$, all experiments arrive at energy densities of $\varepsilon=2-3 \mathrm{GeV} / \mathrm{fm}^{3}$. These numbers are close to the critical values predicted by $Q C D$ lattice calculations for the phase transition and can be considered as a first great success of the experiments. It should be emphasized, however, that rather large uncertainties are involved in both the predicted values and the numbers extracted from the data. Furthermore, the extracted $\varepsilon$-values correspond to the most central collisions and therefore only to a very small fraction of the total cross-section.

An investigation of properties of the formed interaction zone and the search for characteristic signals is therefore rather difficult in experiments with projectiles as small as $\mathrm{O}$ or $\mathrm{S}$ but have already been started. The NA 35 collaboration has used the streamer chamber to measure correlations between produced particles (pions) and to subject the data to an analysis inspired by the early work of Hanbury-Brown and Twiss on measuring the diameter of stars. Since pions are bosons they are positively correlated in momentum space and a measurement of their correlation as a function of momentum difference leads to a characteristic shape. By fitting an ap-

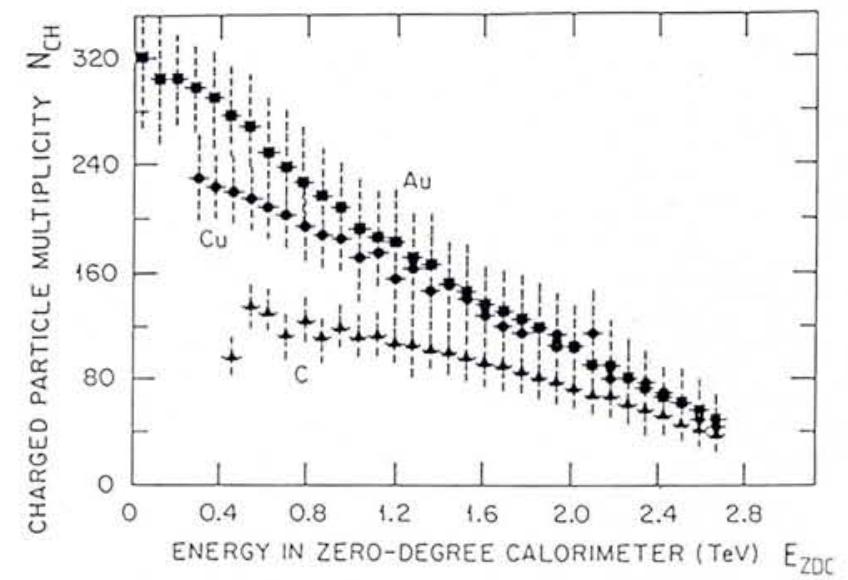

Fig. 3 - Charged particle multiplicity versus energy in the zero-degree-calorimeter $E_{z D S}$ for ${ }^{16} \mathrm{O}$ on different targets at $200 \mathrm{GeV} / \mathrm{A}$ measured by WA 80. 


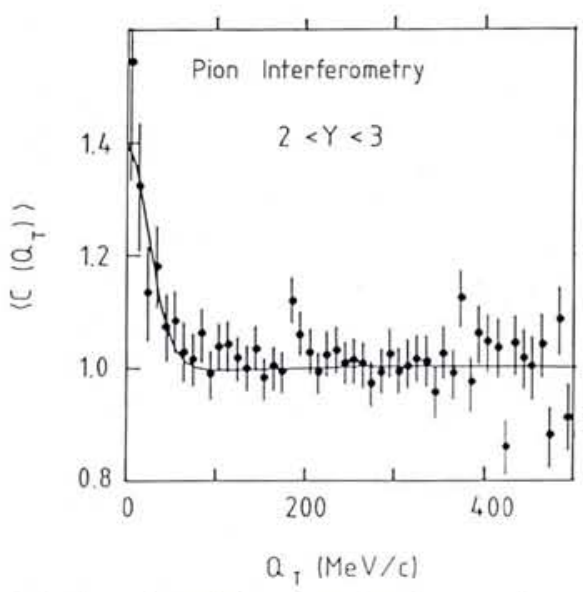

Fig. 4-Correlation function for two pions with transverse momentum difference $Q_{T}$. The curve drawn with the data is a Gaussian fit yielding the radius of the source. Data are ${ }^{16} \mathrm{O}+\mathrm{Au}$ at $200 \mathrm{GeV} / \mathrm{A}$ measured by NA35 in the streamer chamber.

propriate correlation function to the data (Fig. 4), this "pion interferometry" yields information on the size of the emitting source as well the chaoticity and lifetime of the source. In the mid-rapidity region a well defined large source of about $7 \mathrm{fm}$ with large chaoticity is deduced from the data suggesting the formation of a thermalized "fireball".

The WA 80 collaboration has used the photon spectrometer SAPHIR to measure $p_{T}$ spectra of $\pi^{0}$ at mid-rapidity. In models of nucleus-nucleus interactions based on nucleon-nucleon scattering (e.g. Fritiof model) $p_{T}$ spectra should all be similar and close to $p p$ and $p A$ spectra. Deviations of $\mathrm{pA}$ from pp spectra were already observed ten years ago by Cronin et al. Data of O+Au shown in Fig. 5 , however, show that more complicated effects than this "nuclear enhancement" of pA compared to pp occur in the heavy ion data, when impact parameter selection is applied. While peripheral spectra show a behaviour close to $\mathrm{pp}$, with a hard scattering component starting at about $p_{\mathrm{T}} \cong 1.8 \mathrm{GeV} / c$, the central data show a uniform $p_{\mathrm{T}}$ shape for $p_{\mathrm{T}}>$ $0.8 \mathrm{GeV} / \mathrm{c}$ but with a smaller slope than pA data indicating a higher "temperature" of the system.

In view of the fact the critical energy densities are - if at all — only reached for a very small fraction of events, the search for characteristic signals concentrated in these exploratory runs on testing the methods, studying the "normal" behaviour and investigating the background. Direct photons have been identified by WA80, strange particle ratios have been extracted by NA35, but more data processing, a rigorous evaluation of errors and extended Monte Carlo simulations have to be made. For the much discussed $\mathrm{J} / \psi$ suppression, data have been published (Fig. 6) indicating a $20 \%$ suppression in $\mathrm{O}+\mathrm{U}$ compared with $p+U$. Various effects, less spectacular than the quark gluon plasma, are however being discussed to explain this deviation, so that more data and simulation are also needed in this case.

\section{Outlook}

The first series of ultrarelativistic heavy ion experiments at CERN have shown that precision experiments are feasible even in the high multiplicity environment of these reactions and that the search for quark matter is not hopeless. On the contrary, the data on global observables show that the "nuclear stopping power" is high even at 200 $\mathrm{GeV} / \mathrm{A}$ and that energy densities close to the critical values predicted by lattice QCD are reached. First exclusive data also indicate that some type of thermalized reaction zone is formed with properties different from $\mathrm{pp}$ and $\mathrm{pA}$ collisions. Looking for the characteristic signals from the quark gluon plasma seems therefore worthwhile and has in fact be started intensively. It is clear, however, that for this purpose the energy densities and the volume of the region have to be increased. This is possible by using much heavier projectiles. The acceleration of $\mathrm{Pb}$ ion is therefore under consideration which would increase the volume of high energy density by an appreciable factor. Technically, this seems feasible within a time scale of

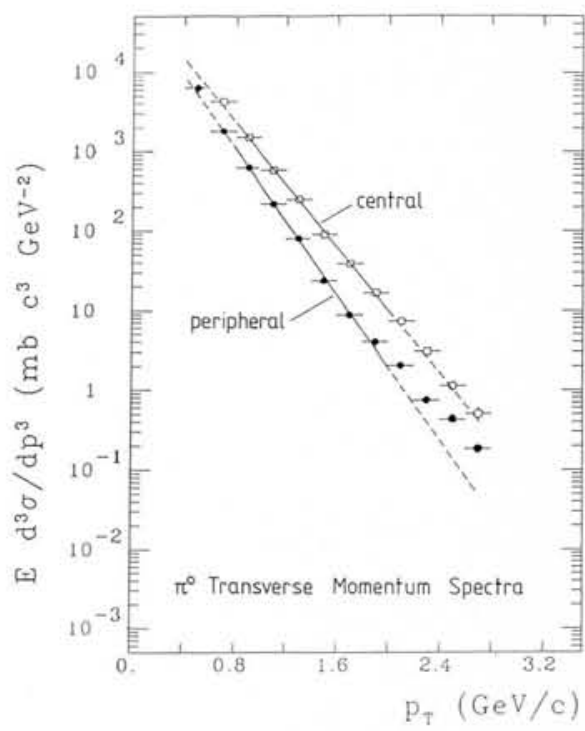

Fig. 5 - Transverse momentum $p_{T}$ spectra of $\pi^{\circ}$ from ${ }^{16} \mathrm{O}+\mathrm{Au}$ at $200 \mathrm{GeV} / \mathrm{A}$ identified by the SAPHIR detector of WA80. The different slopes at medium $p_{T}$ (indicating higher "temperature" for central collisions) and the hard scattering component for high $p_{T}$ in peripheral collisions are clearly seen.

a few years. The community of more than 350 physicists involved in these experiments are therefore hoping that CERN will decide to go ahead.

\section{REFERENCES}

1. 'Quark Matter', Proc. of the 6th Int. Conf. on Ultra-Relativistic Nucleus-Nucleus collisions, Z. Physik C38 (1988).

2. Satz H., Nature 324 (1986) 116.

3. Shuryak E.V., World Scientific Lecture Notes in Physics, Vol. 8 (1988).

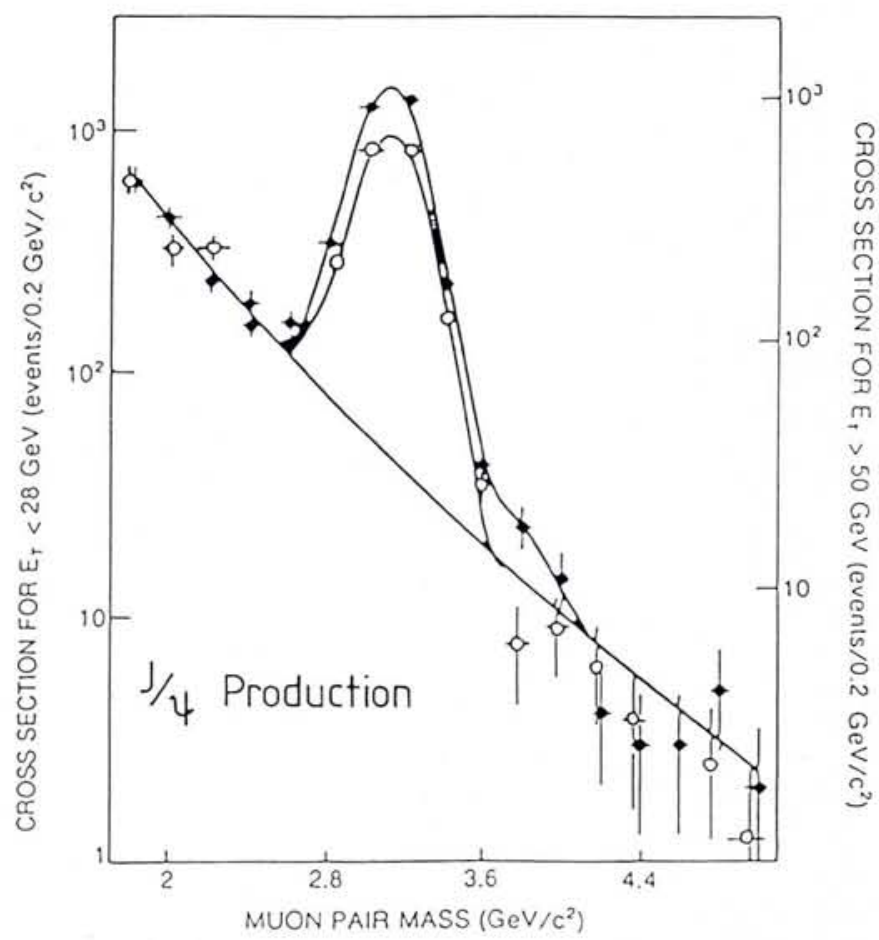

Fig. 6 - Variation of $J / \psi$ production as seen in the $\mu^{+} \mu^{-}$invariant mass spectrum measured by the NA38 collaboration. A suppression of the more central collisions lopen circles) is observed relative to the more peripheral ones (black dots). Note that the background is different in both cases and the data are normalized to the background. 